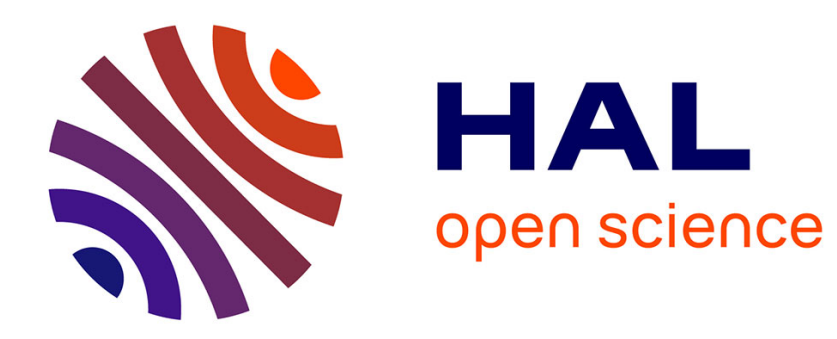

\title{
Les affres de la disparition de la censure
}

Arnaud Esquerre

\section{To cite this version:}

Arnaud Esquerre. Les affres de la disparition de la censure. Communications, 2020. hal-02949834

\section{HAL Id: hal-02949834 \\ https://hal.science/hal-02949834}

Submitted on 22 Dec 2020

HAL is a multi-disciplinary open access archive for the deposit and dissemination of scientific research documents, whether they are published or not. The documents may come from teaching and research institutions in France or abroad, or from public or private research centers.
L'archive ouverte pluridisciplinaire HAL, est destinée au dépôt et à la diffusion de documents scientifiques de niveau recherche, publiés ou non, émanant des établissements d'enseignement et de recherche français ou étrangers, des laboratoires publics ou privés. 


\title{
LES AFFRES DE LA DISPARITION DE LA CENSURE
}

\author{
Arnaud Esquerre
}

Le Seuil | «Communications »

2020/1 n $106 \mid$ pages 147 à 159

ISSN 0588-8018

ISBN 9782021442533

Article disponible en ligne à l'adresse :

https://www.cairn.info/revue-communications-2020-1-page-147.htm

Distribution électronique Cairn.info pour Le Seuil.

(C) Le Seuil. Tous droits réservés pour tous pays.

La reproduction ou représentation de cet article, notamment par photocopie, n'est autorisée que dans les limites des conditions générales d'utilisation du site ou, le cas échéant, des conditions générales de la licence souscrite par votre établissement. Toute autre reproduction ou représentation, en tout ou partie, sous quelque forme et de quelque manière que ce soit, est interdite sauf accord préalable et écrit de l'éditeur, en dehors des cas prévus par la législation en vigueur en France. Il est précisé que son stockage dans une base de données est également interdit. 


\section{Les affres de la disparition de la censure}

La « censure » peut-elle disparaître ? Cette question peut s'entendre au moins de deux manières. Est-il, premièrement, raisonnable de faire disparaître la « censure », et n'est-elle pas nécessaire à l'organisation de tout collectif d'humains vivants? La «censure », défendait à propos du théâtre un de ses historiens, Victor Hallays-Dabot, en 1862, est «pour le gouvernement un instrument de défense contre les agressions des partis » et «pour la société une barrière contre l'envahissement des doctrines perverses ou des peintures immorales $»^{1}$. Cette question, objectera-t-on, n'a plus guère de sens dans un État qui se présente comme démocratique et séparé de toute Église, tel que la France. Elle n'en a plus, tout au moins depuis 1990, lorsque la dernière instance qui pouvait être reconnue institutionnellement comme une commission de " censure ", exerçant son action non plus sur les livres ou les spectacles de théâtre mais sur les films, sous l'appellation de Commission de contrôle, s'est transformée en Commission de classification. Son président d'alors, Jean-François Théry, intitulait Pour en finir une bonne fois pour toutes avec la censure un livre célébrant cette mutation $^{2}$. "Une bonne fois pour toutes », car d'après l'auteur, s'il est certain que la « classification » ne pouvait en aucune manière être qualifiée de « censure », le « contrôle » cinématographique qui la précédait jusqu'alors était abusivement qualifié de «censure ». Il lui aurait, en effet, «manqué une caractéristique de la censure : l'arbitraire » car «les décisions de refus de visa, ou celles qui imposaient des coupures, étaient des décisions administratives susceptibles de recours pour excès de pouvoir devant le Conseil d'État ${ }^{3} »$.

Apparaît alors la seconde manière de comprendre la question initiale : est-il possible de faire disparaître la « censure » dès lors que celle-ci n'est pas reconnue par une institution, mais qu'elle est formulée sous forme d'une accusation et qu'elle peut s'étendre à toute image ou à tout énoncé, y compris non encore exprimé tel un "monologue intérieur » ou un rêve?

J'appelle censure institutionnelle un genre d'actions restreignant la circulation de formes d'expression revendiquées comme telles par une institution, qui 
peut être l'État, un parti politique ${ }^{4}$ ou une Église. La censure institutionnelle, qu'elle soit d'État, partisane ou religieuse, est généralement étudiée à partir des principes de son organisation et des textes censurés, mais il reste peu de traces des discussions des censeurs, qui ne sont guère retranscrites ${ }^{5}$. La censure accusatoire est un genre d'accusation émise par une personne pour s'indigner ou dénoncer des pratiques que ceux qui les réalisent nomment, de leur côté, « choix », « sélection », « modération », « correction », « classification », etc. Cette censure accusatoire a été théorisée depuis le dernier quart du $\mathrm{Xx}^{\mathrm{e}}$ siècle, notamment par Jacques Derrida, Pierre Bourdieu et Judith Butler, et a pu être appelée aussi « censure postmoderne » ou "nouvelle théorie de la censure ${ }^{6}$ ».

Si l'on s'en tient aux conditions d'énonciation, l'« autocensure », elle, peut prendre la forme d'une accusation ou d'une justification exprimée après coup et tournée vers soi-même (« je me suis autocensuré »), ou d'une accusation visant à dénoncer l'action, présente ou future, d'une personne ou d'un collectif empêchant d'exprimer tel message. Toute limitation de la liberté d'expression, qu'elle résulte du respect d'une norme juridique, ou d'un processus de sélection, quelles qu'en soient les raisons, peut conduire à une dénonciation d'《 autocensure ». En France, en 1975, alors que les films pornographiques s'étaient répandus sur les écrans de cinéma et étaient, à ce moment-là, de grands succès, une loi a introduit une taxe élevée qui conduisit, en pratique, à réduire fortement la production de ces films. En janvier 1976, la revue française Cahiers du cinéma s'ouvrait par un éditorial se scandalisant de l'interdiction aux moins de 18 ans des « films pornographiques, pervers et violents (P.P.V.) », démasquant « sous le sourire du libéralisme avancé, la grimace du fascisme ", et prévenant que la loi visait à empêcher les films de se faire et à instaurer l'« autocensure ", c'est-à-dire la « lobotomie des cinéastes ${ }^{7} »$.

\section{Censure institutionnelle.}

Contrairement à ce qu'affirmait l'auteur de Pour en finir une bonne fois pour toutes avec la censure, la « censure » était revendiquée comme telle lorsqu'elle a été mise en œuvre pour les films, tant par l'État que par l’Église, au début $\mathrm{du} \mathrm{Xx}^{\mathrm{e}}$ siècle. Le responsable du Service de contrôle des films était appelé dans les documents administratifs « censeur-chef », et dans le rapport que ce dernier a établi pour rendre compte de la première année de fonctionnement de son service, en 1920, il parle sans réserve d'une action de «censure ", s'en félicitant : " la censure a procédé, sans heurts et sans réclamations notables, et, ce qui est presque paradoxal, à la satisfaction de tous ${ }^{8} »$. De son côté, l'Église catholique a créé en 1934 la Centrale catholique du cinéma et de la radio (CCR) et ce qu'elle appelle une "commission de censure ", composée de prêtres, de religieux et de laïcs, chargée de voir tous les films distribués, et de publier 
ses avis dans les Fiches du cinéma. En 1934, une note interne de la Centrale catholique expliquait, entre autres choses, que des « salles d'actualités appelées "Cinéac" (cinq salles à Paris) se sont engagées envers la Centrale à soumettre à cette commission de censure, chaque semaine, leurs programmes entiers et à $\mathrm{y}$ faire toutes les coupures qu'elle demanderait afin que ces salles puissent être fréquentées par la jeunesse ${ }^{9}$ ».

La « censure » cinématographique de l'État et celle de l'Église catholique s'exerçaient cependant différemment. L'État contrôlait systématiquement tous les films a priori, avant de les autoriser à être distribués, en leur attribuant un visa. Il pouvait, d'une part, exiger des coupes, d'autre part interdire totalement l'accès des spectateurs à un film en refusant de délivrer un visa. Dans les années 1920, il ne distinguait pas de catégories de spectateurs : le film était autorisé ou non pour tous. L'Église catholique, elle, « censurait », c'est-à-dire condamnait ou non les films a posteriori. La Centrale catholique du cinéma et de la radio précisait, dans une note interne de janvier 1937, que « par censure il faut entendre ici non pas la suppression de tel ou tel passage indésirable dans un film, mais le contrôle de tous les films existants et l'application à chacun d'eux d'une note morale ${ }^{10} »$. Mais, outre cette «note morale » guidant les choix de nombreux spectateurs catholiques, l'Église parvenait aussi à exercer une « censure " a priori, comme en témoigne une autre note interne : «le secrétariat de la Centrale est très souvent consulté par des firmes qui lui soumettent le scénario des films avant de le faire réaliser pour éviter une appréciation défavorable de la Centrale, une fois le film réalisé ${ }^{11}$ ». En outre, la " censure » catholique établissait des catégories de spectateurs. La « cotation morale » proposée dans les années 1930 par le journal catholique Choisir délimitait ainsi six catégories de spectateurs :

$\mathrm{P}$ - Le film convient aux enfants des écoles et des patronages.

S - Le film peut être présenté dans les salles familiales.

$\mathrm{T}$ - Le film, sans convenir à toutes les salles familiales, peut être vu en famille.

$\mathrm{R}$ - Le film est à réserver aux personnes formées.

D - Le film n'est pas sans danger, même pour les personnes formées.

$\mathrm{M}$ - Le film est mauvais. ${ }^{12}$

Les premières interdictions locales des films datent de la première décennie du $\mathrm{xx}^{\mathrm{e}}$ siècle, soit la décennie suivant l'invention du cinéma ${ }^{13}$. Or, à la même époque, était élaborée une nouvelle conception de la «censure ». En 1895, année pendant laquelle Louis Lumière réalisait son premier film, La Sortie de l'usine Lumière à Lyon, présenté à Paris le 22 mars à la Société d'encouragement pour l'industrie nationale, Sigmund Freud débutait une réflexion sur les rêves, qui aboutissait à son ouvrage publié quatre ans plus tard, sous le titre de L'Interprétation du rêve. Il y proposait de concevoir la déformation du rêve 
comme le pendant, dans la vie intérieure psychique, de la «censure » dans la vie sociale, exercée sur les ouvrages publiés et la correspondance postale ${ }^{14}$. Le modèle de la «censure » pris par Freud est celui d'une censure politique de l'écrivain qui, redoutant cette dernière, « déforme l'expression de son opinion ${ }^{15}$ », parle par allusions et use de déguisements d'apparence anodins. Cette « censure » au service du « détenteur du pouvoir » traque l'interprétation cachée des écrits, comme la « censure " royale dans l’État monarchique français du XVIII ${ }^{e}$ siècle, cherchant, notamment, à identifier des membres de la Cour dans des personnages de récits à clé (tel Tanastès, conte travestissant la vie amoureuse du $\operatorname{roi}^{16}$ ), et conduisant Diderot à prévenir le lecteur de l'Encyclopédie, à l'article «Encyclopédie » : «On ne sait souvent ce qu'un homme a pensé sur les matières les plus importantes. [...] Ses contemporains même ignorent ses sentiments; et l'on ne doit pas s'attendre que l'Encyclopédie soit exempte de ce défaut ${ }^{17}$. » C'est une telle conception qu'a reprise, dans les années 1950, Léo Strauss, pour qui un écrivain, persécuté et risquant la peine capitale, peut présenter «la vérité sur toutes les questions cruciales $^{18}$ " entre les lignes, ce qui suppose toutefois qu'il existe une nette séparation entre une minorité de lecteurs, formant une élite capable de lire correctement entre ces lignes, et une majorité qui en serait incapable. Cette conception qui met l'accent sur une minorité située au haut d'une hiérarchie sociale, sans être en position de gouverner, peut être aussi inversée, en considérant, comme l'a avancé James Scott ${ }^{19}$, qu'un groupe dominé, situé en bas d'une hiérarchie sociale, peut inventer des manières de faire circuler des messages en les dissimulant, déjouant la loi ou la « censure » imposée par un groupe de dominants. En haut ou en bas, ces groupes minoritaires s'expriment de manière codée pour opposer une résistance à ceux qui ont un pouvoir de suppression de l'accès à un message, ou d'une suppression, partielle ou complète, du message lui-même.

\section{Censure accusatoire.}

Après Mai 68 et jusque dans les années 1980, bien que le dispositif relatif au cinéma repose toujours, pour l'État, sur la délivrance d'un visa, et, pour l’Église catholique, sur une cotation morale, le terme de « censure » n'est progressivement plus assumé, ni par l'un ni par l'autre. Pour l'Office catholique français du cinéma, désormais, "cotation n'est pas censure », est-il expliqué dans un document interne daté du 21 juin 1968. Le comité de cotation de l'Office «n'exerce aucune censure » et, pour autant, son action reste la même : « il visionne, étudie, sélectionne et classe les films afin de conseiller et d'orienter le public vers les meilleurs ${ }^{20} »$. De la même manière, du côté de l'État, le président de la République, Valéry Giscard d'Estaing, déclare, le 27 août 1974, que « les libertés publiques sont et seront minutieusement respectées, plus d'écoutes, 
plus de censure, ni sur les films, ni dans les prisons... », mais, s’interroge la rédaction de la revue Positif en avril 1975, « sur quoi peut bien se fonder cette libéralisation, puisque la Commission de contrôle cinématographique est maintenue, puisque, de tous les arts, le cinéma continue d'être le seul placé sous une pareille tutelle ${ }^{21}$ ?»

Des années 1970 jusqu'aux années 1990, dans un environnement où il n’y a plus de «censure » revendiquée et reconnue institutionnellement comme telle, tant par l'État que par l'Église catholique, il serait «naîf », avertit Derrida en 1990, d'en conclure que la «censure » a disparu ${ }^{22}$. La «censure » se développe sous forme d'accusation mais, étant accusatoire, elle ne désigne plus les mêmes actions que celles qui étaient assumées par l'État et l'Église catholique. Elle est à déceler dans l'intériorité des sujets en tant qu'ils sont situés et pris dans des relations sociales qui s'imposent à eux, et chaque relation sociale, comme chaque intériorité sont suspectes d'être le lieu de l'exercice d'une « censure ». L'extension par Freud de la «censure » exercée par l'État à celle s'exerçant dans l'intériorité des sujets sur leurs rêves est alors reprise et étendue par Pierre Bourdieu. «C'est à condition, écrit Bourdieu, d'apercevoir que le modèle freudien est un cas particulier du modèle plus général qui fait de toute expression le produit d'une transaction entre l'intérêt expressif et la nécessité structurale d'un champ agissant sous forme de censure, que l'on peut rapatrier sur le terrain de la politique, où ils se sont souvent formés, les concepts élaborés par la psychanalyse ${ }^{23}$. » Ainsi s'opère un étonnant mouvement circulaire : conçue à partir d'une conception de la « censure » politique, la théorie de la «censure » à l'œuvre dans les rêves devient à son tour la matrice d'une conception de la « censure » politique. L'agent n'a pas davantage de prise sur ce qu'il exprime qu'il n'en a sur ses rêves, "il n'a même pas à être, en ce cas, son propre censeur, puisqu'il est en quelque sorte une fois pour toutes censuré, à travers les formes de perception et d'expression qu'il a intériorisées et qui imposent leur forme à toutes ses expressions ${ }^{24}$ ». Mais, si la structure exerce une " censure ", il est alors possible de désigner ceux qui sont en position d'imposer les règles organisant cette structure, les « dominants », dans une perspective que l'on peut, cette fois, rapprocher de $\mathrm{Marx}^{25}$, pour qui « la censure est la critique officielle » et « ses normes [...] des normes critiques $^{26} »$.

Cette conception, que l'on peut donc lire comme une hybridation de celles de Marx et Freud, est largement partagée par Derrida, puisque, d'après ce dernier, « il y a censure dès que certaines forces (liées à des pouvoirs d'évaluation et à des structures symboliques) limitent simplement l'étendue d'un champ de travail, la résonance ou la propagation d'un discours ${ }^{27}$ ». Mais d'après Bourdieu, cette « censure » ne crée pas seulement du silence là où quelque chose aurait pu être exprimé si l'individu ne s'était pas censuré, qu'il puisse dire en avoir eu consciemment ou non l'intention, elle peut aussi faire surgir une parole scandaleuse ${ }^{28}$. Or, conteste Foucault à propos de « la trame de mises en 
discours variées, spécifiques et coercitives " autour du sexe au XIX siècle en Europe, il y aurait un effet paradoxal à respecter des normes sociales que l'on traite comme s'il s'agissait de « censure » conduisant au silence, alors qu'elles organiseraient, au contraire, une prolifération de discours relatifs à l'objet visé par ces normes : « une censure massive, depuis les décences verbales imposées par l'âge classique? Il s'agit plutôt d'une incitation réglée et polymorphe aux discours ${ }^{29} »$.

L'idée de la «censure » comme «pouvoir producteur » a été développée, entre autres, par Judith Butler ${ }^{30}$, dont la conception emprunte à Bourdieu l'idée selon laquelle « la censure vise à produire des sujets selon des normes explicites et implicites ${ }^{31} »$. Butler cherche une voie pour caractériser la « censure » entre deux autres conceptions qu'elle rejette : premièrement, " on ne peut jamais entièrement lever la censure qui pèse sur un texte »- conception qui ferait que s'opposer à la « censure », ce serait s'opposer aux conditions de l'intelligibilité ; deuxièmement, « on ne peut jamais entièrement censurer un texte », car le discours excéderait le censeur qui lui impose des limites. Lorsque Butler fait primer la « censure » à l'œuvre dans l'intériorité des sujets sur celle extérieure (juridique), elle oriente l'identification des censeurs vers ceux qui produisent les normes intériorisées, c'est-à-dire les dominants. Toutefois, la « censure implicite » s'exerçant avant tout discours, la question se pose de savoir ce qu'il y a avant la sélection par le tamis de la norme du dicible. À quoi Butler répond qu' « on ne peut le connaître que par l'imagination ${ }^{32}$ ». Dans la perspective de Butler, tous les discours pourraient être probablement acceptables à condition de changer le sens des mots par une opération "performative » : on pourrait tout dire, mais on ne pourrait pas tout signifier. Mais est-il possible de changer le sens des mots? Cela signifierait exclure certaines situations d'énonciation, ce qui permettrait par conséquent d'exclure certains sens de l'espace des sens. Par exemple, le sens de l'«humiliation » serait exclu, et les contextes d'énonciation dans lesquels les mots signifiant une humiliation seraient exclus, pour n'autoriser que les contextes dans lesquels ces mots seraient au contraire glorifiants et rendraient fiers celles et ceux auxquels ils sont adressés ou qui les emploieraient. Le problème est que n'importe quel mot peut être « ressenti » comme humiliant et offensant, dès lors qu'une personne dit le ressentir comme tel participant à la construction du contexte dans lequel le sens du mot varie.

Pour élaborer leurs théories de la censure accusatoire, Derrida et Bourdieu analysent des textes écrits par Kant pour le premier, et par Heidegger pour le second. Mais, d'une part, comme elles plongent dans l'intériorité, ces théories de la censure accusatoire ne sont guère dissociables d'images intérieures entremêlées à un langage intérieur. D'autre part, elles ont été étendues, par d'autres auteurs, aux images, par exemple celles de programmes télévisés destinés à la jeunesse $e^{33}$. 


\section{L'aporie des deux formes de la censure.}

Alors que la «censure » n'est plus revendiquée comme telle par l'État, il reste un examen a priori des images filmées, sous la forme de la «classification », mise en place en 1990, ouvrant la possibilité d'une suppression de l'accès au film pour certaines catégories de spectateurs. Si l'on tient ensemble, d'une part les accusations de «censure " visant l'intériorisation des normes sociales, d'autre part l'exercice d'une «classification » des films permettant une suppression a priori de leur accès, surgit alors une aporie. En effet, dès lors qu'un individu a intériorisé « les formes de perception et d'expression » au point de ne pouvoir exprimer que ce qu'il est légitime d'exprimer, sauf à émettre une "parole scandaleuse », comment est-il possible qu'un film, œuvre collective longue à réaliser, qui nécessite de réunir un financement soumis, pour partie, à des processus de sélection multiples, puisse encore, avant qu'il ne soit distribué dans des salles de cinéma, être examiné par une commission qui décide ou non de supprimer son accès, soit totalement (même si une telle suppression, juridiquement possible, n'est plus pratiquée depuis les années 1980 jusqu'aux années 2010), soit partiellement (aux spectateurs les plus jeunes)? Si les «normes » sociales du dicible et de l'indicible, de ce qui peut être montré et ne peut pas l'être, sont intériorisées par chacun des sujets, pourquoi un État estime-t-il nécessaire de garder une action extérieure sur des formes d'expression avant qu'elles ne circulent dans l'espace public ? Si les réalisateurs de films ont intériorisé des formes de perception et d'expression qui imposent leur forme à toutes leurs expressions, pour reprendre les termes de Bourdieu, pourquoi tous les films ne sont-ils pas autorisés à tous les publics, quand bien même leurs fabricants disent avoir eu cette intention et nullement celle d'émettre une «parole scandaleuse »? Comme l'explique un producteur et réalisateur de films qui conteste, en 2005, dans une lettre à la présidente de la Commission de classification des films, un avis interdisant un film qu'il a produit aux moins de 12 ans :

Lorsque nous produisons un film qui a pour ambition d'être « tous publics », nous avons du mal à évaluer quels peuvent être les risques de « censure » puisqu'il n'existe pas de définition (même vague) ni de classement précis, ni de critères permettant de qualifier une œuvre «tous publics ». Si une telle règle existait, il nous suffirait de les appliquer pour avoir la certitude d'obtenir le visa souhaité ${ }^{34}$.

Une première réponse à ces questions serait que tous ceux qui participent à la réalisation et à la production d'un film n'auraient pas intériorisé les normes sociales aussi profondément que les autres sujets d'un État. Mais la raison pour laquelle ils seraient suspects d'être en capacité de se soustraire aux normes 


\section{Arnaud Esquerre}

sociales en raison de leur activité professionnelle est inexplicable. Une autre piste est que la «classification» ne vise plus tous les spectateurs, ni la suppression du contenu d'un film, comme aux débuts de la censure institutionnelle en France, mais seulement la suppression de l'accès à un film pour des catégories de spectateurs en fonction de leur âge (moins de 12 ans, de 16 ans, ou de 18 ans dans les années 2010). Le problème, alors, ne serait pas que les fabricants de films (en l'occurrence les réalisateurs et les producteurs) n'auraient pas suffisamment intériorisé les normes d'expression applicables à tous, mais celles d'expression applicables aux plus jeunes.

Cela nous conduit à devoir examiner de plus près le principe au fondement de la « censure ", qu'elle soit une action reconnue par une institution (censure institutionnelle), ou une accusation (censure accusatoire), et qui est de prêter des effets aux formes d'expression, qu'il s'agisse de discours ou d'images. La manière dont des auteurs comme Bourdieu, Derrida ou Butler ont traité la question des effets sociaux des énoncés et des images s'est limitée à deux grands effets : l'effet d'autorité et l'effet performatif, effets auxquels peuvent être associées les expressions du mépris, de l'humiliation et de l'offense, d'une part; de l'honneur, de la fierté et de la reconnaissance, d'autre part.

Cependant, la «classification" des films ne s'appuie ni sur des effets d'autorité ni sur des effets performatifs à l'aune desquels la « censure »a été repensée sous forme d'accusation mais elle s'appuie, d'une part sur la peur et le traumatisme censés être induits par des images d'actes violents, d'autre part sur la jouissance sexuelle et la perturbation supposées être produites par des images d'actes sexuels; les deux pouvant se croiser, notamment à propos d'images présentant des actes à la fois sexuels et violents et qualifiées, par certains membres de la commission, de sadiques ou de sadomasochistes. Ce qui gît au cœur des avis de la Commission de classification des films, et qui justifie une suppression de l'accès à un film pour une catégorie de spectateurs en fonction de leur âge, c'est leur "sensibilité », qui peut être "heurtée » ou «troublée ", c'est-à-dire l'effet des images sur leur intériorité. La « classification » des images filmées ne cible pas leur interprétation cachée, mais leur interprétation ostensible. N'ayant pas, par principe, d'accès direct à cette intériorité d'un spectateur absent, les membres de la Commission de classification élaborent un spectateur projeté à partir de leur proximité avec des mineurs, et de leurs propres souvenirs d'enfance : chacun dit avoir en mémoire des images qu'il « n'aurait pas dû voir » lorsqu'il était lui-même enfant, cette expérience collective étant présentée comme une exception singulière, dont chaque membre serait chargé d'éviter qu'elle ne se reproduise dans les futures générations ${ }^{35}$.

Or l'interprétation des images conduisant à estimer leurs effets sur l'intériorité de spectateurs projetés varie avec le temps. Cette variation est repérable à quelques décennies d'intervalles, à propos d'un même film. Soit, par exemple, Les Nuits de Dracula de Jesus Franco Manera ${ }^{36}$. Après l'avoir visionné 
le 20 novembre 1970, la sous-commission de contrôle cinématographique a émis l'avis suivant :

Sans être réellement très effrayant, il semble que ce film doive être assorti d'un visa d'interdiction aux mineurs de 13 ans en raison du caractère traumatisant que peuvent revêtir certaines scènes pour un jeune public, notamment les visions fréquentes et répétées de Dracula et de ses adeptes se livrant à leur distraction favorite : sucer le sang de leurs victimes et celles, non moins écœurantes où les ennemis de Dracula entreprennent de l'éliminer ainsi que toute la secte en les transperçant ou leur tranchant la tête dans leur cercueil.

Réexaminé en décembre 1997 et en janvier 1998, et en dépit du fait que certains membres de la sous-commission aient estimé que « l'impact nocif sur la sensibilité des plus jeunes des spectateurs de multiples images délibérément effrayantes (sans parler d'un climat constamment angoissant) subsiste ", l'avis de la Commission de classification a été d'autoriser désormais le film à tous les publics.

En outre, l'interprétation des effets des images filmées sur l'intériorité des « jeunes » spectateurs diffère selon les spectateurs « adultes ». Lors de l'examen d'un film en 2015, les membres du comité de classification sont partagés au point de proposer quatre sortes d'avis :

2 interdiction[s] - 12 ans avec avertissement : film décrivant toutes les étapes d'une histoire amoureuse avec de très belles images, rien de gratuit ou complaisant. Euvre romantique avec un montage dans lequel les scènes de sexe érotiques sont diluées de manière pudique. Rien de pornographique. Drogue et violence sont présent[e]s.

6 interdiction[s] - 16 ans : œuvre profondément romantique, douce, fragile et non provocatrice. Film sur la fin d'une relation amoureuse, les souvenirs, les regrets et le spleen de la vie de couple. Scènes érotiques trop explicites, sexe non simulé, scènes avec utilisation de drogues diverses ne s'adressant pas à un jeune public.

2 interdiction[s] - 16 ans avec avertissement : scènes de sexe très explicites. Difficulté de tracer une frontière entre ce qui relève de l'érotisme et ce qui peut être taxé de pornographique. Pour ce film il existe bien un récit, une histoire.

2 interdiction[s] - 18 ans : nombreuses scènes de sexe non simulées et de drogue. Mélodrame aussi érotique que pornographique, le film ne peut s'adresser à un public mineur ${ }^{37}$.

À ces divergences parmi les membres d'une commission s'ajoute le fait que des adultes peuvent se mobiliser pour contester des avis de la Commission de 
classification et obtenir une restriction de l'accès aux films. La contestation porte sur le fait que des membres nommés par l'État pour « classifier » des films n'auraient pas correctement intériorisé les normes permettant de déterminer ce qu'il serait légitime que des «enfants » ou des «adolescents » voient ou ne voient pas. Ainsi, le film Love de Gaspard Noé ayant été interdit aux moins de 16 ans assorti d'un avertissement (" Les très nombreuses scènes de sexe sont susceptibles de heurter la sensibilité du public »), une association, Promouvoir, qui a pour objet de « défendre la dignité de la personne humaine et de protéger les mineurs à travers la "promotion des valeurs judéo-chrétiennes" ", d'où son nom $^{38}$, en a contesté le visa auprès des juges. À la question «Quel serait le système idéal selon vous d'attribution d'un visa de classification ? ", l'avocat de l'association, André Bonnet, répond qu' « il n'existe pas d'idéal dans ce domaine, car la question fondamentale est d'ordre intérieur : il faut connaître les plus jeunes, les aimer, et avoir gardé suffisamment de fraîcheur soi-même pour anticiper ce qui peut leur faire du mal, sans tomber dans le puritanisme, ni dans la naïvetéte ${ }^{39} »$. Mettant en avant auprès du tribunal administratif «l'existence de scènes à caractère pornographique, incluant des mineurs, et tournées de la manière qui les rend le plus crédibles possible », le recours en suspension formulé par l'avocat de l'association Promouvoir conclut que «c'est justement l'environnement "léché" du film qui peut conférer à ce dernier un effet encore plus dévastateur sur les mineurs ${ }^{40}{ }^{\text {}}$. Rejoignant l'avis de Promouvoir, le juge des référés du tribunal administratif de Paris, dans son ordonnance du 31 juillet, a considéré que plusieurs scènes de Love « comportent une représentation des relations sexuelles qui, sans toutefois caractériser des scènes à caractère pornographique et nonobstant la volonté artistique du réalisateur, sont de nature à heurter la sensibilité des mineurs ${ }^{41}$ » et, donc, de nature à justifier une interdiction du film aux moins de 18 ans, décision confirmée par le Conseil d'État le 30 septembre 2015.

S'agissant du cinéma, l'abandon progressif de la censure institutionnelle, reconnue comme telle par l'État et l'Église catholique après Mai 68, a ouvert l'espace en France à une censure accusatoire visant l'intériorisation de "normes » par les sujets, exprimée notamment par Bourdieu. Cette censure accusatoire est une extension du modèle de la «censure » appliqué aux rêves par Freud, modèle provenant lui-même d'une conception de la censure institutionnelle telle qu'elle était pratiquée dans des États monarchiques européens avant le $\mathrm{xx}^{\mathrm{e}}$ siècle. Une telle « censure » cherchait à déceler les interprétations cachées dans les textes, ce qui supposait, pour la contourner, des auteurs écrivant de manière codée, et des lecteurs capables de déchiffrer des codes.

Or, le modèle d'une censure accusatoire intériorisant les normes imposées par les structures du champ et par les «dominants » à l'intérieur de ce champ ne permet de comprendre ni le maintien d'une instance de «classification » des films dans le prolongement d'une censure institutionnelle, ni le fait qu'une 


\section{Les affres de la disparition de la censure}

telle instance n'autorise pas tous les films à tous les publics, ni le fait que les avis de cette instance puissent être parfois contestés par des associations se présentant comme défendant la jeunesse et la dignité humaine. Il est peut-être naïf de conclure que la «censure » a disparu, mais il est peut-être encore plus naïf de penser que l'intériorisation des normes est telle qu'elle a lissé les formes d'expression. Car même des cinéastes expérimentés peuvent avoir une hésitation sur ce qu'il faut dire ou ne pas dire, montrer ou ne pas montrer. Et cette incertitude est tout autant partagée par ceux qui doivent interpréter les films en vue de donner un avis recommandant ou non de les autoriser à tous les spectateurs ou d'en supprimer l'accès à certains d'entre eux. Le problème n'est pas tant que les images filmées produisent des effets sur les jeunes, mais qu'elles produisent des effets différents sur tous les spectateurs - ceux qui les réalisent comme ceux qui sont en charge d'en supprimer l'accès.

\section{Arnaud Esquerre \\ arnaud.esquerre@ehess.fr}

CNRS-Institut de recherche interdisciplinaire sur les enjeux sociaux (IRIS)

\section{NOTES}

1. Victor Hallays-Dabot, Histoire de la censure théâtrale en France, Paris, E. Dentu, 1862, p. 208.

2. Jean-François Théry, Pour en finir une bonne fois pour toutes avec la censure, Paris, Cerf, 1990.

3. Ibid., p. 27.

4. Des membres du Parti ouvrier unifié polonais siégeaient ainsi avec des représentants de l'État et des professionnels du cinéma dans les commissions de validation des films en Pologne dans les années 1970. Ania Szczepanska, «La guillotine et le pressoir », 1895. Mille huit cent quatre-vingt-quinze, 69, 2013, p. 70-95.

5. Parmi les exceptions, on peut relever les transcriptions des réunions de commission de validation des films en Pologne, étudiées (de 1972 à 1983) par Ania Szczepanska, ibid.

6. Matthew Bunn, « Reimagining Repression. New Censorship Theory and After », History and Theory, 54 , p. 25-44.

7. Jean-Louis Comolli, "Éditorial : la censure libérale avance », Cahiers du cinéma, 262-263, janvier 1976, p. 5-8.

8. Rapport de M. Paul Ginisty sur le fonctionnement du service de contrôle des films cinématographiques en 1920, Archives nationales, F/21/8665.

9. «Quelques idées pour la circulaire à nosseigneurs (NN. SS.) les évêques. L'activité de la Centrale catholique du cinéma et de la radio depuis sa création ", décembre 1934, Archives de l'Église de France, 2CE5.

10. Centrale catholique du cinéma et de la radio, note de janvier 1937, Archives de l'Église de France, 2CE5.

11. "Quelques idées pour la circulaire à NN. SS. les évêques », art. cité.

12. Chanoine Joseph Reymond, "Notre critique des films », Choisir. Vivre c'est choisir, 31, 30 avril 1933, p. 1.

13. Kenneth Garner et Richard Abel, « Regulating a Risky Business : Film, Censorship, and Public Safety in Prewar France, 1909-1914 », Yale French Studies, 122, "Out of Sight : Political Censorship of the Visual Arts in Nineteenth-Century France », 2012, p. 160-185. 


\section{Arnaud Esquerre}

14. Sigmund Freud, L'Interprétation du rêve (1899), Paris, PUF, « Quadrige », 2010, p. 177-179.

15. Ibid., p. 178.

16. Robert Darnton, De la censure. Essai d'histoire comparée, Paris, Gallimard, 2014.

17. Article «Encyclopédie », Encyclopédie, t. V, 1755 ; cité par Antoine Lilti, L'Héritage des Lumières. Ambivalences de la modernité, Paris, EHESS/Gallimard/Seuil, 2019, p. 310.

18. Léo Strauss, La Persécution et l'Art d'écrire (1952), Paris, Gallimard, « Tel », 2009, p. 55.

19. James Scott, La Domination et les arts de la résistance. Fragments d'un discours subalterne (1990), Paris, Éditions Amsterdam, 2009.

20. «Qu'est-ce que l'OCFC (Office catholique français du cinéma)? ", 21 juin 1968, Archives de l’Église de France, 2 CE 1008.

21. «Les infortunes de la liberté », par la Rédaction, Positif. Revue de cinéma, n 168, avril 1975, p. 76.

22. Jacques Derrida, «Chaire vacante : censure, maîtrise et magistralité », Du droit à la philosophie, Paris, Galilée, 1990, p. 347.

23. Pierre Bourdieu, «Censure et mise en forme» (1982), Langage et pouvoir symbolique, Paris, Seuil, « Points », 2001, p. 344.

24. Ibid., p. 345.

25. Matthew Bunn, « Reimagining repression », art. cité.

26. Karl Marx, « Remarques à propos de la récente instruction prussienne sur la censure » (1843),

Euvres. Philosophie, Paris, Gallimard, 1982, p. 111.

27. Jacques Derrida, "Chaire vacante ", op. cit., p. 348.

28. Pierre Bourdieu, «Censure et mise en forme », op. cit., p. 344.

29. Michel Foucault, Histoire de la sexualité 1. La Volonté de savoir, Paris, Gallimard, 1976, p. 47.

30. Judith Butler, "Censure implicite et puissance d'agir discursive " in Le Pouvoir des mots. Discours de haine et politique du performatif (Excitable Speech, Routledge, 1997), Paris, Éditions Amsterdam, 2004, p. 201-252.

31. Ibid., p. 209.

32. Ibid., p. 216.

33. Voir, par exemple, l'usage de la conception de la «censure » de Bourdieu par Jean-Matthieu Méon, "Contrôle concerté ou censure ? L'euphémisation du contrôle public des médias et sa légitimation », Raisons politiques, 2005, 17/1, p. 149-160.

34. Archives du CNC.

35. Arnaud Esquerre, Interdire de voir. Sexe, violence et liberté d'expression au cinéma, Paris, Fayard, 2019, p. 183-192 ; "Classez ce film que les enfants ne sauraient voir », Terrain, 72, 2019, p. 24-41.

36. Dossier Les Nuits de Dracula de Jesus Franco Manera, archives du CNC.

37. Dossier Love de Gaspard Noé, archives du CNC.

38. Entretien avec André Bonnet, Première, disponible sur www.premiere.fr, 9 juillet 2015.

39. Ibid.

40. Dossier Love de Gaspard Noé, archives du CNC.

41. Tribunal administratif de Paris, juge des référés, $n^{\circ}$ 1511962/9, ordonnance du 31 juillet 2015.

\section{RÉSUMÉ}

Les affres de la disparition de la censure

On peut distinguer deux formes de «censure » : une censure institutionnelle, reconnue comme telle par une institution, comme l'État ou une Église, et qui a été pratiquée en France pour le cinéma jusque dans les années 1970 ; une censure accusatoire, qui s'est développée largement à partir de Mai 68 et de la disparition de la censure institutionnelle, et qui a été théorisée notamment par Pierre Bourdieu, Jacques Derrida et Judith Butler. Toutefois, il est resté, en France, une action de « classification » des 


\section{Les affres de la disparition de la censure}

films au début du Xxi ${ }^{\mathrm{e}}$ siècle exercée par l'État, qui est difficilement compatible avec les théories d'une censure accusatoire.

MOTS-CLÉS : censure, liberté d'expression, cinéma, classification

\section{SUMMARY}

The Woes of the Disappearance of Censorship

There are two forms of "censorship": institutional censorship, recognized as such by an institution, such as the State or the Church, and practiced in France for movies until the 1970s; an accusatory censorship, which developed largely from May 68 onward and in the wake of the disappearance of institutional censorship, and which has been theorized especially by Pierre Bourdieu, Jacques Derrida, and Judith Butler. However, in France there remains a "classification" of movies at the beginning of the $21^{\text {st }}$ century conducted by the State, and it is hardly compatible with theories of the accusatory censorship.

KEYWORDS : censorship, free speech, cinema, classification

\section{RESUMEN}

Las tribulaciones de la desaparición de la censura

Hay dos formas de "censura" : la censura institucional, reconocida como tal por una institución como el Estado o la Iglesia, y practicada en Francia para el cine hasta la década de 1970 ; la censura acusatoria, que se desarrolló en gran parte a partir de mayo del 68 y de la desaparición de la censura institucional, y que ha sido teorizada especialmente por Pierre Bourdieu, Jacques Derrida y Judith Butler. Sin embargo, a comienzos del siglo XXI permanece en Francia una operación de "clasificación" de las películas, ejercida por el Estado, que es difícilmente compatible con las teorías de la censura acusatoria.

PALABRAS CLAVES : censura, libertad de expresión, cine, clasificación 